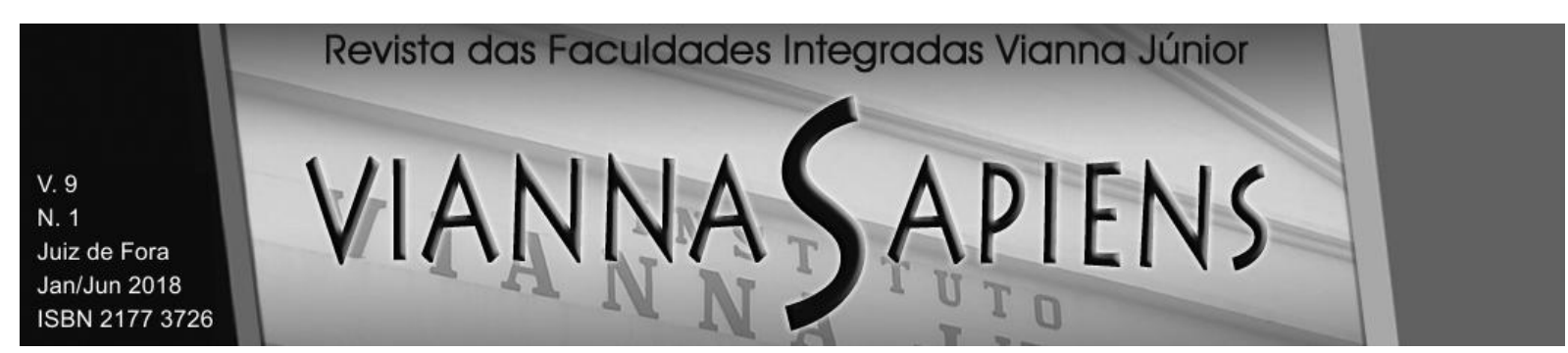

\title{
AS ROTINAS DE ORÇAMENTO COMO LÓCUS PARA ESTUDOS SOBRE AS ROTINAS ORGANIZACIONAIS: Um ensaio teórico
}

DOI: 10.31994/rvs.v9i1.361

Samantha Luiza de Souza Broman ${ }^{1}$ Sandra-Regina da Rocha Pinto ${ }^{2}$

\section{RESUMO}

As rotinas organizacionais passaram a ser vistas como generativas e com um potencial para a mudança contínua. O presente ensaio teórico alega que as rotinas do orçamento empresarial (budgeting) representam uns lócus adequado para estudos sobre a dinâmica das rotinas (Routine Dynamics), relacionando esses temas, sob a ótica processual e a lente dos Estudos Organizacionais. Os orçamentos são processos que integram o sistema de controle e planejamento das empresas e interagem com rotinas adjacentes. Destaca-se que existem sugestões para que se estude 'como' os orçamentos são usados. Assim, cabe considerar como são percebidas as combinações e adaptações das rotinas orçamentárias, havendo uma dinâmica interna subjacente e como podem ser percebidos como úteis. Os orçamentos também tratam do sub-tema dos artefatos, pois são considerados como um instrumento de gestão preponderante. Portanto, sugere-se uma agenda de pesquisa empírica com as abordagens deste debate e o enfoque na ecologia das rotinas orçamentárias.

\footnotetext{
${ }^{1}$ Mestre em Administração de Empresas pela PUC-Rio - Brasil; email sambroman@hotmail.com http://orcid.org/0000-0001-5239-8629

${ }^{2}$ Doutora em Educação, Mestre em Administração de Empresas pela PUC-Rio - Brasil; email sanpin@iag.puc-rio.br; http://orcid.org/0000-0002-5121-4231
} 
PALAVRAS-CHAVE: ROTINAS ORGANIZACIONAIS.

ORÇAMENTO EMPRESARIAL. PERSPECTIVA PROCESSUAL.

\section{INTRODUÇÃO}

A rotina organizacional vem sendo estudada no campo dos estudos organizacionais pela lente da prática e no campo da estratégia pela lente das capacidades (PARMIGIANI; HOWARD-GRENVILLE, 2011).

A partir das publicações de Feldman (2000) e Feldman e Pentland (2003), os estudos sobre as rotinas organizacionais tomaram um novo rumo, trazendo evidências empíricas de que as rotinas são dinâmicas por possuírem um potencial endógeno para mudanças contínuas. Logo, as rotinas não são apenas estáveis ou inflexíveis (FELDMAN, 2003; FELDMAN; PENTLAND, 2003; PARMIGIANI; HOWARD-GRENVILLE, 2011). Desse modo, a corrente que estuda as partes constituintes das rotinas pela lente da prática aponta que elas são 'generativas' porque suas ações podem gerar mudanças na própria forma como são desempenhadas ou podem ser mantidas; e são 'emergentes' porque existem em sua execução, em sua situação específica (HOWARD-GRENVILLE; RERUP, 2017; RERUP; FELDMAN, 2016).

Há, atualmente, um movimento acadêmico que sugere que as novas pesquisas sobre as rotinas organizacionais sejam realizadas a partir de uma perspectiva processual, de forma a contribuir com a compreensão sobre a dinâmica interna das rotinas [Routine Dynamics] e sobre como interagem rotinas organizacionais múltiplas/simultâneas: a ecologia das rotinas (FELDMAN et al., 2016). Ou seja, sugere-se estudar as rotinas não apenas isoladamente, mas considerar as influências mútuas entre rotinas que são desempenhadas de forma conjunta; compreender melhor como ocorrem as padronizações nessas interações e como se reestabelecem as tréguas entre ostensivos conflitantes (FELDMAN, 2016; FELDMAN; PENTLAND; D'ADDERIO; LAZARIC, 2016; HOWARD-GRENVILLE; RERUP, 2017). 


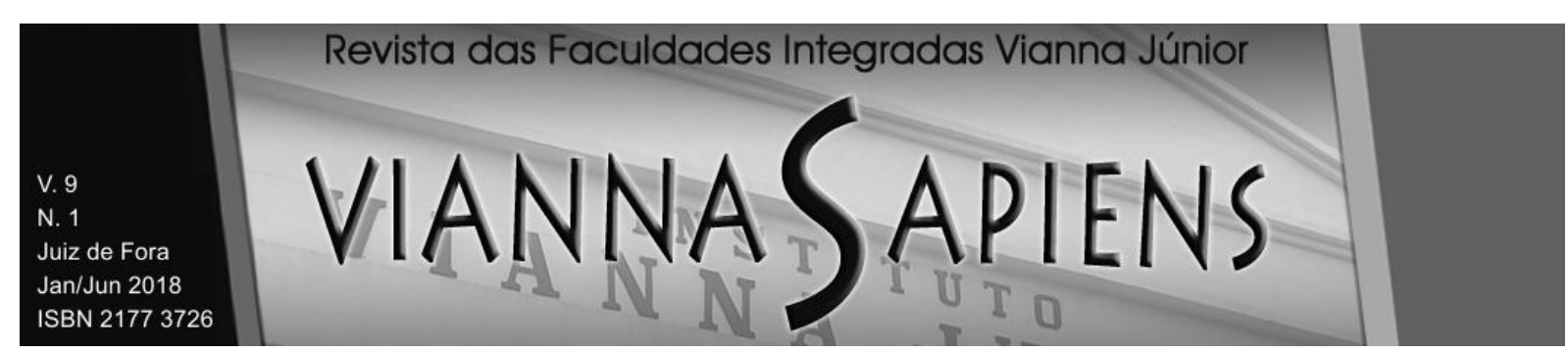

O orçamento empresarial foi um dos temas sugeridos para serem investigados como rotina 'em execução' (FELDMAN et al., 2016 p.506) quando esses autores mencionaram que: "os padrões mais frequentemente identificados pelos pesquisadores são relacionados às tarefas organizacionais que estão sendo realizadas por meio de rotinas como: selecionar [hiring], orçar [budgeting], precificar [pricing], planejar [planning]...”.

Segundo Feldman (2016), a perspectiva processual passa a dar maior ênfase nas rotinas em contínua execução - continuity of becoming e menos foco nas rotinas como 'entidades'. A autora traz a ação para o foco dos estudos organizacionais sobre rotinas, alegando que a sua natureza é constitutiva e transcende dualismos. Por essa perspectiva, o que vem sendo tratado em termos de aspectos performativo e ostensivo das rotinas, passa a ser abordado em termos de suas execuções e padronizações - performing and patterning.

O presente trabalho é um ensaio teórico, tendo em vista que se constitui de uma natureza interpretativa e reflexiva, apresenta elementos subjetivos, assim como a proposta de novas perguntas para se apreender a realidade (MENEGHETTI, 2011). Ademais, as elaborações e ideias apresentadas, com base em reflexões, intuições e conhecimentos acumulados sobre os temas em questão, buscam abrir "perspectivas para caminhos futuros" (BORTERO; MACEDO; SETTE, 2011 p. 342) que contemplem 'como' as ações e padronizações das rotinas orçamentárias influenciam a forma como são percebidas, desempenhadas (havendo uma dinâmica interna) e se relacionam com outras rotinas (pessoas e artefatos), sob uma perspectiva processual.

Assim sendo, apresentam-se reflexões sobre possíveis convergências entre os temas das Rotinas Organizacionais e o Orçamento Empresarial. Para endossar essa discussão, foi possível identificar na literatura de orçamentos pistas para que os orçamentos sejam estudados sob o olhar qualitativo dos estudos organizacionais, pois Merchant (1998 p.332) argumenta que cabe buscar o entendimento sobre "como fazer" o orçamento (e não apenas como planejá-lo) e Libby e Lindsay (2010) alegam que problemas [críticas] sobre os orçamentos podem estar relacionadas com a forma como os orçamentos são usados. 


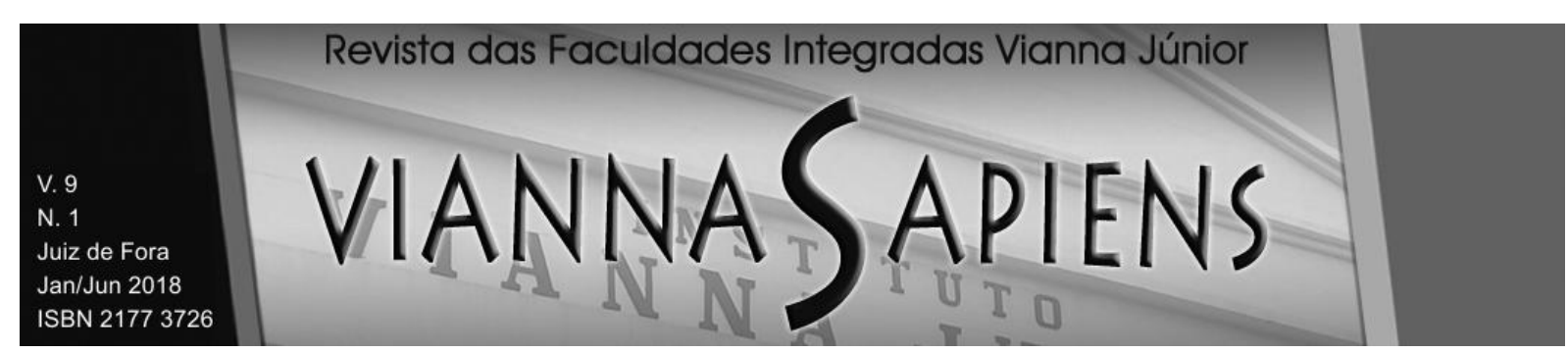

Uma primeira coerência entre a perspectiva processual/prática adotada pelos estudos sobre as Rotinas Organizacionais e o tema dos Orçamentos Empresariais pode ser observada pelo fato de que as publicações do campo da contabilidade também abordam os orçamentos na forma da sua execução contínua, em gerúndio: 'budgeting' (BECKER et al. 2016; HANSEN; VAN DER STEDE, 2004; LIBBY; LINDSEY, 2010; MERCHANT, 1981). Essa abordagem condiz com a afirmação de Langley e Tsoukas (2010) sobre o crescente uso do 'ing' para estudar fenômenos organizacionais no ambiente global e de rápidas mudanças.

Na perspectiva de orçamentos como processos, Merchant (1998) ilustra que o processo orçamentário requer que haja compartilhamento de informações, objetivos, prioridades, necessidade de recursos, restrições e riscos através da organização, cuja coordenação das informações percorre fluxos top-down, bottom-up ou mesmo laterais. Ou seja, o budgeting integra um sistema do processo de planejamento das organizações. (MERCHANT, 1981, 1998). Do mesmo modo, Mucci, Frezzati e Dieng (2016 p. 286) explicam que: "o orçamento deve ser visto como um processo". Enquanto isso, Sponem e Lambert (2010) afirmam que o orçamento deve ser compreendido como um conjunto de práticas e Ekholm e Wallin (2000) mencionam um planejamento contínuo e um processo de controle.

Para o uso da leitura sobre rotinas orçamentárias, a partir da lente que considera as rotinas como emergentes, generativas e possuidoras de uma dinâmica interna (HOWARD-GRENVILLE; RERUP, 2017), o presente trabalho toma como referência: 1. a afirmação de Libby e Lindsay (2010) de que as organizações optam por adaptar suas práticas orçamentárias, sem abandonar o orçamento tradicional (anual); 2. os estudos que identificaram que os orçamentos continuam integrando os propósitos de planejamento e controle das empresas (ELKHOLM; WALLIN, 2011; FREZZATI, 2005; PIETRZAK, 2013; SPONEM; LAMBERT, 2010), mesmo após as críticas da década de 90 sobre o orçamento tradicional/anual poder ser ineficiente no ambiente de incertezas, dispendioso, gerar pouco valor, entre outras (HOPE; FRASER, 2003b).

Pesquisas mais recentes trazem a percepção de utilidade tanto do tradicional orçamento anual-fixo, quanto das formas alternativas de orçar, tratadas como 
orçamentos 'flexíveis' por Ekholm e Wallin (2011), podendo ser complementares. Desse modo, Réka, Stefan e Daniel (2014) entendem que os orçamentos modificados (recriados em novos tipos de orçamento), como o rolling forecast se adequam melhor a um ambiente mais moderno.

Em uma reflexão sobre como essas rotinas de orçamento com foco no curto prazo e que buscam ser mais descentralizadas, como o rolling forecast (HOPE; FRASER, 2003b), são traduzidas do seu aspecto ostensivo para serem incorporadas na prática em si (em uma contínua execução), destaca-se o papel dos indivíduos que podem tomar iniciativas para adaptar a forma de desempenhá-las para retomar os propósitos orçamentários ('effortful accomplishment') (PENTLAND; RUETER, 1994) e aprimorá-los. Tal fenômeno pode ser paralelo ou não às atualizações do conteúdo das informações orçamentárias (e valores). Quanto às influências externas, a literatura de orçamentos aponta que os impactos variam de acordo com cada organização, pois conforme Padoveze e Taranto (2009) exemplificam: até mesmo um fator como o 'clima' pode ser muito influente em uma empresa e em outra não. Cabe destacar que também existem pesquisas posteriores sobre as funções e razões dos orçamentos e sobre suas tipologias (EKHOLM; WALLIN, 2011; MUCCI; FREZZATI; DIENG, 2016; SPONEM; LAMBERT, 2010).

No caso do Brasil, pode-se intuir que as volatilidades político-econômicas enfatizadas por notórias crises, escândalos e mesmo as flutuações em termos de inflação, câmbio, PIB e etc. tem sido impactantes para os orçamentos, agravando um cenário de urgências que podem exigir não só atualizações dos conteúdos orçamentários como também intensificar as dinâmicas e interações entre as diferentes áreas das empresas (as rotinas múltiplas) para decisões compartilhadas, renovar objetivos e tréguas, assim como novas padronizações (patterning) para orçar (FELDMAN, 2016).

Diante do exposto, o presente ensaio almeja despertar um interesse sobre como a análise das rotinas orçamentárias pode contribuir com lacunas atuais na literatura de Rotinas Organizacionais. Paralelamente, ao trazer o tema de orçamentos para o campo dos estudos organizacionais, acredita-se que discussões sobre a convergência dos temas e novos estudos empíricos, possam descortinar 
expansões da teoria, por meio de problematizações que contribuam para além do preenchimento das lacunas identificadas (ALVESSON; SANDBERG, 2011), trazendo questões como a percepção da utilidade e de aspectos favoráveis e/ou desfavoráveis relacionados com a conduta orçamentária.

Nesse sentido, com a ressalva que, nas rotinas de orçamento, todos são corresponsáveis pelo seu desenvolvimento (RÉKA; STEFAN; DANIEL, 2014), cabe uma reflexão para o fato de que o potencial endógeno para mudanças nas rotinas poder ser deflagrado, também, por integrantes de outras rotinas que sejam adjacentes às rotinas de orçamento e inter-relacionadas com estas.

Outra ponte entre os temas de Orçamento Empresarial e as Rotinas Organizacionais refere-se ao papel dos artefatos na dinâmica das rotinas organizacionais (FELDMAN; ORLIKOWSKI, 2011; HOWARD-GRENVIELLE; REROUP 2017; PARMIGIANI; HOWARD-GRENVILLE, 2011), pois no caso da literatura dos orçamentos, o 'budget' é também apontado como um instrumento de gestão preponderante (FREZATTI; NASCIMENTO; JUNQUEIRA; RELVAS, 2011; PIETRZAK, 2013; SPONEM; LAMBERT, 2010).

Os artefatos são tratados como entrelaçados às rotinas organizacionais, com a indicação de que a materialidade é um importante fator na forma como as rotinas são realizadas (FELDMAN; ORLIKOSKI, 2011). A literatura das rotinas destaca os artefatos que desempenham um papel de script das rotinas - uma proxy do ostensivo da rotina (ex: SOP - Standart Operational Procedures)) e/ou os artefatos pelos quais é possível estudar sobre as rotinas (ex: workflow/logs) (FELDMAN; PENTLAND; D'ADDERIO; LAZARIC, 2016). Todavia, a literatura de orçamento empresarial, explica que esse instrumento de gestão - 'o budget' é uma ferramenta para o gerenciamento do controle (RÉKA; STEFAN; DANIEL, 2014) e mecanismo de direção [decisão], pelo qual se pode corrigir o percurso (EKLHOM; WALLIN, 2000), entre outras aplicações. Ou seja, se por um lado o orçamento - budgeting é um processo, pode-se compreendê-lo também como 'o meio e o produto' dinâmico da própria rotina e da interação entre diferentes rotinas, no papel de seu instrumento - 0 budget. 
Portanto, o presente debate procura uma reflexão sobre as dinâmicas das rotinas organizacionais em sua ecologia, na perspectiva processual e visa a reforçar o valor da oportunidade de se estudar esse tema a partir das rotinas de orçamento. Desse modo, sugere-se que sejam realizados estudos empíricos que busquem investigar como os profissionais de planejamento/orçamento percebem as interações entre as rotinas de orçamento - budgeting e outras rotinas organizacionais. Futuros trabalhos podem também pesquisar sobre o papel do próprio instrumento de gestão - o budget na dinâmica das rotinas.

Cabe destacar que o presente debate adota uma postura neutra em relação às distintas correntes de estudos dos orçamentos, seja a linha que confirma a utilidade percebida do budgeting e que evidencia a manutenção proeminente de suas práticas (LIBBY; LINDSAY, 2010) ou a corrente de críticas à eficiência do processo orçamentário anual e à forma como é usado em avaliações de performance dos indivíduos (HOPE; FRASER, 2003b).

Este artigo traz, primeiramente, uma breve revisão da literatura recente sobre as Rotinas Organizacionais para mostrar caminhos propostos pela corrente da perspectiva processual. Em seguida, são apresentadas algumas definições e uma parte da evolução da literatura de Orçamento Empresarial, demonstrando como este tema permanece interessante tanto para a academia, quanto para as organizações. Por fim, apresenta o debate teórico a respeito de como o orçamento empresarial adequa-se, de forma relevante, para uma pesquisa qualitativa sobre as rotinas, para o campo dos estudos organizacionais, ilustrando como esses temas se relacionam e podem expandir as conceituações vigentes. Nas considerações finais há uma sugestão para que futuros estudos empíricos sobre as rotinas (pela perspectiva processual) adotem o contexto dos orçamentos. 


\section{FUNDAMENTAÇÃO TEÓRICA}

\subsection{Rotinas Organizacionais}

No avanço dos estudos sobre as rotinas organizacionais, a concepção das rotinas como 'vivas' e não apenas como 'inertes' foi explicada por Feldman (2000; 2003). A autora identificou que as rotinas podem ser continuamente modificadas/recriadas, à medida que os indivíduos que as desempenham encontram resultados diferentes do esperado ou quando discordam sobre como executá-las (HOWARD-GRENVILLE; LANGLEY; TSOUKAS, 2016). Essa abordagem estuda a dinâmica interna das rotinas, enquanto as abordagens decorrentes da lente da economia evolucionária (NELSON; WINTER, 1982) teorizam as rotinas como 'caixas pretas' e como habilidades da organização ligadas ao DNA da organização, da qual surgiram os pilares para a corrente de estudos das capacidades dinâmicas (FELDMAN, 2016).

Assim, com a lente teórica de Giddens (1984) e Bourdieu (1992), sobre a dualidade entre a agência e a estrutura, Feldman e Pentland (2003) explicam os dois aspectos da mesma rotina que são mutuamente constituintes: o aspecto performativo, que representa as rotinas em execução em suas situações específicas e o aspecto ostensivo que se refere à ideia abstrata da rotina. Esse ideal da rotina pode ser compreendido diferentemente por diferentes pessoas, pois mesmo que seja transcrito em procedimentos (scripts), as interpretações podem variar (FELDMAN; PENTLAND, 2003; PARMIGIANI; HOWARD-GRENVILLE, 2011)

Desse modo, os achados de Feldman $(2000,2003)$ e conceituações de Feldman e Pentland (2003) semearam o caminho de estudos sobre dinâmica das rotinas - Routine Dynamics em seu papel não apenas para a estabilidade, mas também em seu potencial endógeno para mudança (PARMIGIANI; HOWARDGRENVILLE, 2011). Tal fato é consequência de que as pessoas geram novos padrões de ação, mesmo que não haja influências externas para isto ou, por outro lado, podem manter seus padrões de ação em resistência às influências externas (FELDMAN, 2016). Feldman (2003) aponta que dentre os seus estudos empíricos 
desenvolvidos na State University, nos quais foram identificadas modificações nas rotinas, a rotina de orçamento não obteve uma mudança que havia sido desejada top-down, tendo em vista uma inconsistência entre o entendimento das pessoas sobre a rotina e como a organização atuava.

Feldman (2016) esclarece que a execução e a padronização são mutuamente constituintes na dinâmica das rotinas e explica que o aspecto performativo das rotinas é bem compreendido como as ações das pessoas nas situações específicas, mas que cabe reforçar que o aspecto ostensivo (o ideal abstrato da rotina) é inseparável da rotina. Isto porque ainda existem interpretações que podem não discernir entre o aspecto ideal da rotina e os artefatos como procedimentos operacionais escritos (SOP - Standard Operational Procedures). Nesse sentido, Howard-Grenville e Rerup (2017) também mencionam que recentes trabalhos têm destacado a importância de não se confundir o aspecto ostensivo com os artefatos, pois o aspecto ostensivo não pode ser encapsulado.

Segundo Howard-Grenville e Rerup (2017) e Feldman (2016), a perspectiva processual das rotinas é consistente com a especificidade das rotinas (aspecto performativo) e com a definição apontada por Feldman e Pentland (2003, p. 95) de "padrões repetitivos e reconhecíveis de ações interdependentes realizadas por vários atores". Assim, cabe explorar os mecanismos que se manifestam nas situações de persistência dos padrões e/ou mudanças nas ações e/ou novas padronizações (HOWARD-GRENVILLE; RERUP, 2017).

Feldman (2016, p.24) ainda alega que há uma natureza das rotinas orientada para processos e sugere que se explore "mais profundamente a ontologia processual das rotinas, passando da ênfase nas características das rotinas como 'entidades' para mais ênfase nas rotinas em 'execução contínua' - continuity of becoming". Assim, a autora sugere o foco na ação das rotinas para: transcender-se aos dualismos (em partes separadas da rotina) como: 1. a estabilidade e a mudança ou 2. as citadas dificuldades/divergências de compreensão entre os aspectos ostensivo-performativo (FELDMAN; PENTLAND, 2003) - 'mente e corpo'; e observar-se que a relação das rotinas considera não apenas pessoas, mas também materialidades, emoções, históricos, poder e tempo. 


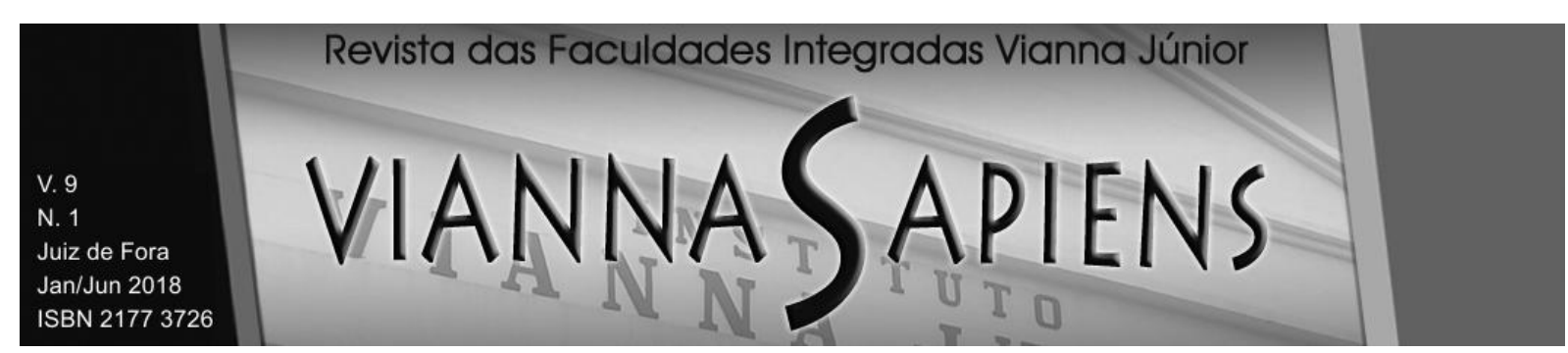

Além disso, o uso dos artefatos (tecnológicos ou não), que participam do dia a dia das rotinas, seja como proxy do ideal das rotinas ou como meio para investigálas (como exemplo um workflow ou os registros de um sistema) também despertam o interesse para compreender como estão entrelaçados à dinâmica das rotinas, bem como para melhor compreender o universo sociomaterial (FELDMAN et al., 2016; FELDMAN; ORLIKOWSKI, 2011)

Assim sendo, Howard-Grenville e Rerup (2017) apontam que há campo para que futuros trabalhos aprofundem sobre a perspectiva processual das rotinas organizacionais, para compreensão da dinâmica de como as padronizações se formam e evoluem partir das ações; como múltiplas rotinas se relacionam (retomando as naturezas das tréguas vs conflitos) - a ecologia das rotinas; e como a temporalidade e a espacialidade moldam e interferem nas rotinas.

Feldman et al. (2016 p.506) sugerem que se estude as rotinas orçamentárias (budgeting) em sua execução contínua. Inclusive, a autora (2003) já estudou essas rotinas e evidenciou a relevância do alinhamento/desalinhamento entre como os objetivos da rotina são entendidos em seu ideal abstrato (aspecto ostensivo) e a sua execução, pois, neste caso, as mudanças que eram desejadas pela diretoria não aconteceram.

\subsection{Orçamento Empresarial}

O orçamento empresarial é um dos temas da contabilidade gerencial (FREZATTI; NASCIMENTO; JUNQUEIRA, 2011; PIETRZAK, 2013) e representa um plano financeiro para um determinado período, para o qual estima-se previsões de vendas, de custos, de produção, de fluxo de caixa e outros, pelo qual busca-se coordenar o que deve ser feito para atingi-lo (RÉKA; STEFAN; DANIEL, 2014).

Os orçamentos se mantêm com proeminência na prática organizacional e permanece sob o interesse acadêmico, mesmo após as críticas que surgiram em relação ao orçamento tradicional, a partir da década de 90 - a Era da Informação (HOPE; FRASER, 2003a). De uma forma geral, Libby e Lindsay, (2010 p. 67) apontam que o orçamento "continua a desempenhar um papel chave nos sistemas 
de controle gerencial das organizações". Artigos sobre o tema e aqueles que buscaram responder e atualizar sobre essas críticas ao orçamento tradicional encontram-se nos periódicos da contabilidade. Segundo a revisão de literatura feita por Gomes, Lavarda e Torrens (2012), o periódico Accounting, Organizations and Society concentrou a maior parte das publicações entre 2000 e 2009. O periódico Managment Accounting Research e outros como o Social Sciences também dedicaram atenção ao tema.

Ensina Badem (2016) que o início do orçamento empresarial se deu há quase um século, nos EUA, enquanto o orçamento público tenha originado na Inglaterra. Mesmo que seja difícil precisar o momento do início do orçamento empresarial, seu uso se intensificou após a segunda guerra (BADEM, 2016; RÉKA; STEFAN; DANIEL, 2014).

O budgeting é explicado por Merchant (1981 p. 813) como um sistema, sendo uma "combinação de fluxo informacional e processos e procedimentos administrativos que, normalmente, integram parte do sistema de controle e do planejamento da organização". No entanto, o escopo do orçamento nas organizações é amplo, pois suas conceituações, funções e papéis são explicados com amplo envolvimento com a estratégia, com o planejamento, com o controle e com o dia a dia das organizações.

Desse modo, a rotina de orçar - budgeting é mencionada por alguns autores por apresentar múltiplas funções (HANSEN; VAN DER STEDE, 2004), compor um 'sistema de controle' (EKHOLM; WALLIN, 2000; LIBBY; LINDAY, 2010) e ser um importante instrumento de gestão - o budget (FREZZATI et al., 2011; MUCCI; FREZZATI; DIENG, 2016; PIETRZAK, 2013). Nesse sentido, Merchant (1998) aponta que a organização colhe benefícios pelo orçamento integrar o processo de desenvolver planos da organização, pois conduz as pessoas a pensar e debater suas ideias sobre o futuro da organização, de forma que se comprometem com as metas da organização. Pode-se perceber que, por um lado, é definido como um processo orçamentário (LIBBY; LINDAY, 2010), que contém diferentes funções em diferentes momentos (MUCCl; FREZATTI; DIENG. 2016), mas, também é 
mencionado como um instrumento de gestão (um artefato) importante para as organizações.

Sponem e Lambert (2010 p. 161) alegam que o orçamento deve ser "apreendido como um conjunto de práticas". Após um trabalho de tipologia de estilos orçamentários, esses autores apontam que a multiplicidade de funções do orçamento não é de todo problemática. As múltiplas funções do orçamento também apresentam variações conforme os autores, pois segundo Mucci, Frezatti e Dieng (2016) elas se sobrepõem e há uma longa história acadêmica sobre isso. As funções/razões do orçamento denotam dois momentos: ex ante e ex post (MUCCI, FREZATTI; DIENG, 2016). Também em relação a essas duas etapas, Hansen e Van der Stede (2004) discorrem sobre 4 ensejos para o orçamento, subdivididas em: curto prazo (planejamento operacional e avaliação de desempenho); e longo prazo (comunicação de metas e formação de estratégia). Por outro lado, Ekholm e Wallin (2011) dividem as funções do orçamento em um processo de planejamento (planejamento, coordenação, alocação de recursos e determinação dos volumes operacionais) e na função de diálogo (comunicação, criação de consciência e motivação). A interação destas temporariedades e funções pode ser compreendida pelo uso de indicadores e metas a serem cumpridos (Budget Targets), pois estes são aplicadas como parâmetros para avaliações da performance, conforme citado na função de 'diálogo' (EKHOLM; WALLIN, 2011) e na tipologia do estilo de 'diagnóstico' (SPONEM; LAMBERT, 2010).

Desta forma, Kihn (2011 p.4) definiu as metas orçamentárias como as "estimativas/previsões e planos financeiros de resultados futuros, que foram acordados com a gerência, em termos do que se espera deles para coordenar as atividades de diferentes partes da organização, ao longo do tempo". Desse modo, o montante (valor de verbas) que é alocado para as ações futuras das áreas é considerado como autorizado para ser usado pela gerência da área.

O orçamento empresarial tradicional é o orçamento anual, explicado por Merchant (1998) como aquele que se inicia cerca seis meses antes do fim do ano fiscal e que é completado nos últimos dois meses do ano. Este orçamento é chamado de 'fixo' por Ekholm e Wallin (2011). Na década de 90, esse orçamento 
tradicional-anual (projetado para o exercício fiscal seguinte) foi criticado porque não seria eficiente em um ambiente inconstante e competitivo, por consumir muito tempo de trabalho, ser custoso, entre outros (EKHOLM; WALLIN 2000, 2011; LIBBY; LINDAY, 2010). Também foi criticado o uso das remunerações variáveis concedidas aos gestores com base em metas do orçamento anual fixo, tendo em vista que os objetivos pessoais do indivíduo podem interferir no seu trabalho e no resultado da organização (HOPE; FRAZER, 2003A).

Essas críticas foram discutidas, no Beyound Budgeting Roundtable (BBRT), que reuniu participantes de diversas organizações e teve adesão de diversos países, com base em exemplos de algumas empresas que eliminaram a forma de orçar anualmente e buscaram outras formas de gestão e de descentralizar as informações (RÉKA; STEFAN; DANIEL, 2014), incluindo análises e tendências, nas quais o calendário de foco no ano é substituído por um contínuo monitoramento - rolling forecast e considera rubricas importantes como: vendas, custos, lucros e fluxos de caixa (HOPE; FRASER, 2003a).

No entanto, apesar dessas críticas, estudos mais recentes indicam que as empresas não planejam encerrar as suas atividades do orçamento anual - fixo (anual budgets), mas combiná-lo e adaptá-lo com formas alternativas de orçar, como o AAB - activity-based budgeting e o próprio Rolling Forecast, mais descentralizados (EKHOLM; WALLIN 2000, 2011; LIBBY; LINDAY, 2010; PIETZRAK, 2013). Ekholm e Wallin (2011) chamaram essas formas alternativas de orçar de 'orçamentos flexíveis' e, em seus achados, evidenciaram a percepção de pode haver uma complementariedade entre os orçamentos anuais - fixos e esses 'orçamentos flexíveis', de forma que essas práticas não são rivais entre si. Ou seja, na acepção do papel do orçamento, Ekholm e Wallin (2011) alegam que as críticas aos orçamentos 'fixos' não se sustentaram, à medida que passaram a coexistir com os orçamentos 'flexíveis', modificáveis em menores intervalos de tempo (variable, flexible, revised and rolling budgets).

A partir da perspectiva dos orçamentos como processos e procedimentos (MERCHANT, 1981), pode-se considerar que os orçamentos flexíveis sejam formas alternativas de orçar decorrentes de variações/adaptações do tradicional orçamento 
anual, pois, segundo Libby e Lindsay (2010 p. 67), as empresas "planejam e tomam passos para aprimorar seus sistemas orçamentários e superar algumas das críticas comuns". Além disso, esses autores alegam que os problemas dos orçamentos são relacionados com a forma "como os orçamentos são usados e não por uma questão de orçamentos serem inerentemente falhos".

Portanto é possível observar que há espaço na literatura para que se estude os orçamentos sob uma perspectiva processual, constituída de ações consecutivas e contínuas. Nesse sentido, Ekholm e Wallin (2000; 2011) também apontam que a prática dos orçamentos flexíveis, como o 'orçamento contínuo' [o Rolling Forecast] possibilita que os tomadores de decisões na organização captem os sinais das mudanças ambientais exógenas à organização e aprenda sobre elas. Estes autores apontam que cabe novas explorações sobre a percepção da utilidade dos orçamentos nas organizações. Desse modo, pode ser interessante investigar se a possibilidade de os profissionais aprimorarem/modificarem a forma como desempenham suas rotinas orçamentárias influencia a percepção de sua utilidade e valor do orçamento.

A respeito desses fatores exógenos - ambientais, que tendem a influenciar os planos financeiros das organizações, cabe destacar a situação do Brasil, que, se, por um lado, o país desenvolveu melhor as práticas orçamentárias após o plano Real (a partir de 1994), com uma estabilização do cenário que era altamente inflacionário e o diferenciava de outros países (LEITE; CHEROBIM; SILVA; BUFREM, 2008), é possível intuir que o recente ambiente volátil político econômico deste país, com variabilidade de fatores como inflação, câmbio, PIB, desemprego, impeachment presidencial e outros, ilustra como significativas incertezas ambientais podem a reforçar a necessidade de frequentes revisões dos orçamentos e, inclusive, da compreensão sobre 'como' os orçamentos se mantém percebidos como úteis para a organização. 


\section{DISCUSSÃO}

Nos estudos organizacionais, o tema das rotinas organizacionais aborda como ocorrem as padronizações, como as rotinas são executadas e como são modificadas endogenamente, tendo em vista que há uma dinâmica interna das rotinas e que múltiplas rotinas se afetam mutuamente (FELDMAN et al., 2016). A convocação é para estudos na interação entre diferentes rotinas, assim como as imbricações entre as rotinas e seus artefatos de trabalho, em uma perspectiva processual.

As rotinas são definidas como: "padrões reconhecíveis e repetitivos de ações interdependentes realizadas por múltiplos atores" (FELDMAN; PENTLAND, 2003, p. 95). Esse conceito continua condizendo com o papel original das rotinas de resolver conflitos e estabelecer tréguas entre os envolvidos, mas se coaduna com a explicação de que as rotinas possuem potencial para mudanças e transformações contínuas. Isto tendo em vista uma perspectiva processual, pela qual estuda-se como mudanças nas práticas são implementadas nas organizações, sem deixar de considerar que as práticas são sociomateriais (LANGLEY; TSOUKAS, 2010). Notase, assim, que há um interesse para que os estudos sobre a dinâmica interna das rotinas contemplem as inter-relações entres rotinas múltiplas e como se dá o papel dos artefatos usados e desenvolvidos no dia a dia das rotinas (FELDMAN et al., 2016; FELDMAN; ORLIKOWSKI 2011; HOWARD-GRENVILLE; RERUP, 2017; PARMIGIANI; HOWARD-GRENVILLE, 2011).

Por sua vez, é possível admitir que o orçamento empresarial pode ser explorado sob a perspectiva da dinâmica das rotinas [Routine Dynamics], uma vez que os orçamentos se configuram como um processo/um conjunto de práticas (MERCHANT, 1981; SPONEM; LAMBERT, 2010). Ademais, há um interesse da própria literatura de orçamentos para que se investigue sobre 'como' os orçamentos são desempenhados (FREZATTI, 2005; LIBBY; LINDSAY, 2010). Além disso, sob a ótica das funções do orçamento, tem-se que elas não são estáticas posto que são interdependentes. Em síntese, pode-se concordar com Mucci, Frezatti e Dieng (2016 
pag. 298) quando afirmam que: "o orçamento consiste em um processo dinâmico dentro do Sistema de Controle Gerencial da empresa, e deve ser visto como tal".

Para fundamentar a argumentação de que os temas Orçamento Empresarial e Rotinas Organizacionais se relacionam pode-se considerar que o cenário de evolução e transformação nas rotinas de orçamento condiz com as evidências de Feldman (2000; 2003) sobre a agência nas rotinas organizacionais (GIDDENS, 1984) e com a afirmação de Pentland e Reuter (1994) sobre os participantes das rotinas precisarem se esforçar para conseguir realizar suas rotinas com sucesso diante das variações que ocorrem ('effortfull accomplishment').

Assim sendo, uma análise da dinâmica interna das rotinas orçamentárias pode contribuir com a compreensão sobre 'como' essas rotinas são modificadas e avançar nas teorizações sobre 'a ecologia das rotinas' (FELDMAN et al., 2016; HOWARD-GRENVILLE; RERUP, 2017). A observação das características de interação e compartilhamento com outras rotinas organizacionais (MERCHANT, 1998), poderá favorecer a percepção da existência de um espaço para expandir as noções sobre como outras rotinas (adjacentes) podem ser deflagradoras do potencial da mudança das rotinas. Ou seja, as rotinas organizacionais impactam e são impactadas entre si e, à medida que despertam o potencial de mudança, exigem uma renovação das tréguas entre os envolvidos de forma que as padronizações e a execução sejam mutuamente constituintes (lembrando que a perspectiva processual transcende dualismos). Nesse sentido, Réka, Stefan e Daniel (2014 p. 575) lembram que dentre as abordagens do orçamento ocorre uma 'negociação', na qual "cria-se um ambiente onde todos são responsáveis pela preparação do orçamento".

Pode-se citar, como exemplo, que dentre as atividades da primeira dimensão da função do orçamento anual-fixo, apontada por Ekholm e Wallin (2011 p.152) como constituintes do "processo de planejamento (planejamento, coordenação, alocação de recursos, determinação de volumes operacionais)", as rotinas de previsão de vendas da organização, demandam modificações nas informações que são atualizadas e trocadas com o ambiente externo, de maneira que podem provocar mudanças consecutivas na forma como o orçamento integra alguma nova configuração dessas estimativas. Esse fato contempla a necessidade de que as 
diferentes áreas envolvidas estejam em consenso, pois pode (tende a) ocorrer que diferentes indivíduos (de diferentes áreas) tenham seus objetivos prioritários diferentes; queiram estabelecer folgas nos valores orçados, podendo provocar conflitos. A própria literatura de orçamentos aborda problemas com os 'slacks', que se referem às reservas sublineares que os gerentes preservam em suas verbas (VAN DER STEDE, 2000). Nesse sentido Hope e Fraser (2003a; 2003b) alertam sobre a possibilidade do conflito entre os objetivos pessoais/parciais dos gerentes atrelados às suas avaliações de desempenho e do resultado organizacional.

Nesse sentido, a interdependência do fluxo de informações e decisões conjuntas entre aqueles que desempenham as rotinas de orçamentos e aqueles que desempenham outras rotinas (MERCHANT, 1998) podem denotar que as múltiplas rotinas sejam um 'gatilho' para mudanças em uma ou outra rotina (deflagradas por indivíduos que desempenham rotinas diferentes, mas interligadas). Adicionalmente, é possível conjecturar que esses gatilhos e inter-relações, assim como a dinâmica interna das rotinas, podem revelar maneiras de prevenção a possíveis maus usos do orçamento ou da descrença de seu papel no conjunto do sistema de controle gerencial da organização.

Assim, considerando a constatação de que a rotina de orçamento empresarial - budgeting tem persistido de forma relevante nas organizações (EKHOLM; WALLIN, 2000, 2011; LIBBY; LINDSAY, 2010; PIETRZAK, 2013; RÉKA, STEFAN; DANIEL, 2014; SPONEM; LAMBERT, 2010), acredita-se ser possível que estudos empíricos sobre as rotinas orçamentárias aprofundem conhecimentos sobre 'como' e 'por que' se desenvolvem as variações na forma de orçar (as combinações entre as práticas dos orçamentos flexíveis, realizados ao longo do ano, e o tradicional orçamento anual, por exemplo). Além disso, pode-se buscar a melhor compreensão sobre 'como' o orçamento pode manter-se percebido como útil e 'quais' aspectos podem ser favoráveis e/ou desfavoráveis à forma como os orçamentos são usados.

Outro ponto convergente entre os estudos sobre as rotinas organizacionais e as rotinas orçamentárias, refere-se ao sub-tema dos artefatos, visto que orçamentos também são chamados de instrumentos de gestão, que formalizam objetivos e 
orientam o plano estratégico, comunicam e compartilham informações (EKHOLM; WALLIN, 2011; MUCCI, FREZATTI; DIENG, 2016; REKA, STEFAN; DANIEL, 2014; PIETRZAK, 2013; SPONEM; LAMBERT, 2010).

Cabe destacar que essa dupla abordagem sobre o orçamento empresarial ser tanto um processo, quanto um artefato, pode corroborar com a concepção de que os aspectos performativo e ostensivo das rotinas são mutuamente constituintes (HOWARD-GRENVILLE; RERUP, 2017; PARMIGIANI; HOWARD-GRENVILLE, 20111). Adicionalmente, considerar o orçamento como um artefato resultante da rotina em si (para uso futuro) pode expandir o entendimento sobre o(os) papel(is) dos artefatos nas rotinas, uma vez que o 'budget' se distingue, de certa forma, dos exemplos de artefatos já mencionados pela literatura de rotinas como: os procedimentos padrões escritos (SOP standard operational procedures) e os workflows/Logs citados por Feldman (2016). Em outras palavras, o aspecto ostensivo do orçamento denota uma formalização de parâmetros (rubricas e valores) que delimitam e direcionam como será a atuação futura nas rotinas adjacentes ao longo do ano e base para análise de variações. Essa concepção se aproxima das definições de Kihn (2011) (p. 5) que "as metas orçamentárias demonstram como os recursos são alocados internamente na organização e podem servir como uma autorização formal para um gerente gastar um montante específico em determinada atividade".

Diante do exposto, é possível afirmar que a perspectiva processual para estudar o orçamento empresarial como lócus de rotina organizacional pode ser julgada adequada para elucidar a compreensão da dinâmica interna das rotinas, suas influências em rotinas adjacentes e vice-versa, além de contribuir com entendimentos sobre a percepção da utilidade e de como é desempenhada a rotina orçamentária. 


\section{CONSIDERAÇÕES FINAIS E PESQUISAS FUTURAS}

As transformações nas formas de desempenhar as rotinas de orçamentos nas últimas décadas corroboram com acepção da dinâmica das rotinas, visto que os orçamentos anuais persistem, e que existem evidências sobre as práticas orçamentárias serem combinadas e poderem ser aprimoradas.

Assim como Kihn (2011) concluiu que o processo orçamentário pode gerar entendimentos diferentes sobre os propósitos e metas orçamentárias, face a questões de subjetividade e posições dos gerentes, cabe refletir o quanto uma investigação empírica qualitativa pode aprofundar em: 'como' uma padronização das ações de elaboração dos orçamentos e dessas metas (ou aprovação ou divulgação) podem influenciar essas diferentes interpretações; 'como' são percebidas as interações entre as rotinas organizacionais; e 'como' a dinâmica interna das rotinas de orçamento empresarial torna-se relevante para a prática orçamentária das organizações. Logo, acredita-se que as rotinas de orçamento são apropriadas para as discussões sobre as lacunas indicadas por Feldman et al. (2016) e Howard-Grenville e Rerup (2017) a respeito da busca pela melhor compreensão sobre a ecologia das rotinas, em uma perspectiva processual, que olha para as rotinas em execução contínua, não apenas como 'entidades' fixas.

Desta forma, abre-se uma agenda de pesquisa para que se investigue, qualitativamente, o tema das rotinas organizacionais nos lócus das rotinas de orçamento empresarial, com o enfoque nas análises trazidas no presente trabalho. Ou seja, sugere-se a investigação sobre como os profissionais envolvidos percebem as interações entre as rotinas de orçamento - budgeting e as outras rotinas organizacionais, podendo abordar, também, o papel do próprio instrumento de gestão - o budget.

Pode-se intuir que o cenário brasileiro seja frutífero para pesquisas empíricas sobre a dinâmica e a ecologia das rotinas de orçamento empresarial, tendo em vista que o ambiente político-econômico instável, pode intensificar a necessidade de as organizações revisarem seus orçamentos com frequência e, consequentemente, surgirem imprevistas interações entre diferentes rotinas organizacionais. Até mesmo 
no caso do orçamento que é realizado anualmente, poucos meses de trabalho significa um período que já pode apresentar inúmeras variações do macro ambiente. Tome-se como um exemplo, os escândalos de corrupções que vêm sendo investigados e divulgados desde 2014 e que têm afetado o cenário econômico e as premissas orçamentárias.

\title{
BUDGETING ROUTINES AS A LOCUS FOR STUDIES ON ORGANIZATIONAL ROUTINES: A theory essay
}

\begin{abstract}
Organizational routines have come to be seen as generative and with a potential for continual change. The present theoretical paper finds that budgeting routines represent an adequate locus for studies in routine dynamics; it relates these themes in terms of both process and organizational studies standpoints. Budgeting refers to processes that integrate a firm's control and planning system and interact with adjacent routines. Several suggestions have been advanced in relation to studying just 'how' budgets are used. Thus, we must consider how the combinations and adaptations of budgeting routines are perceived, given their underlying internal dynamics, and how we may perceive them as being useful. Considered a key management tool, budgets also deal with the sub-theme of artifacts. Therefore, we propose an empirical research agenda, including the various approaches to this debate and focusing on the ecology of budget routines.
\end{abstract}

\section{KEYWORDS: ORGANIZATIONAL ROUTINES. BUSINESS BUDGET. PROCEDURAL PERSPECTIVE.}




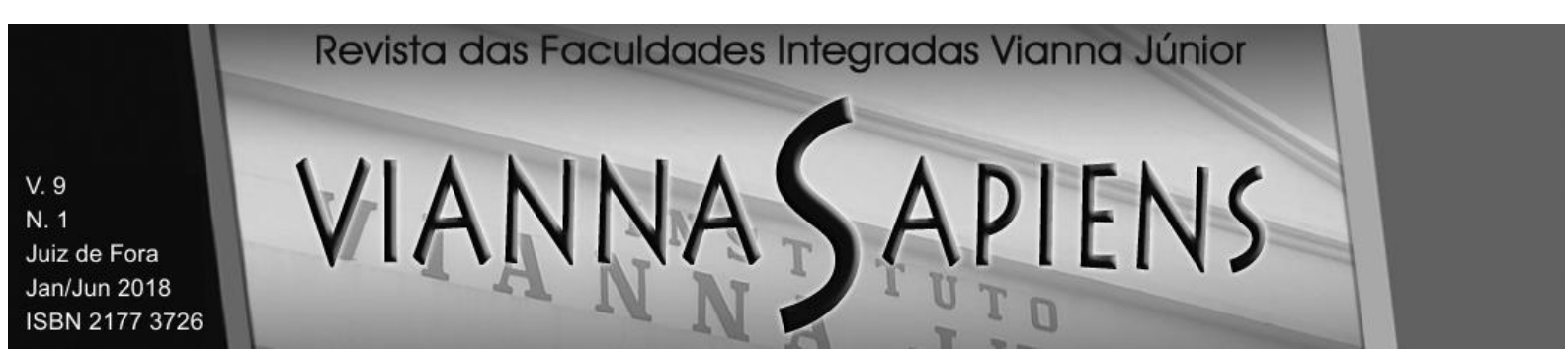

\section{REFERÊNCIAS}

ALVESSON, M.; SANDBERG, J.. Generating research questions through problematization. Academy of management review, 36(2), 247-271, 2011. DOI:10.5465/AMR.2011.59330882

BADEM, A. The origin of term budget for business enterprises: the development of business budgeting from beginning to the 1940 S. Journal of Suleyman Demirel University Institute of Social Sciences, 24(2), 1-28, 2016.

BERTERO, B. Réplica 2 - o que é um ensaio teórico? Réplica a Francis Kanashiro Meneghetti / What is a theoretical essay? Response to Francis Kanashiro Meneghetti. Revista De Administração Contemporânea, (2), 338, 2011. doi:10.1590/S1415-65552011000200012

BOURDIEU, P. The logic of practice. Stanford University Press.1990.

EKHOLM, B.; WALLIN, J. Is the annual budget really dead?. European Accounting Review, 9(4), 519-539, 2000. DOI:10.1080/09638180020024007

EKHOLM, B.; WALLIN, J.. The Impact of Uncertainty and Strategy on the Perceived Usefulness of Fixed and Flexible Budgets. Journal of Business Finance \& Accounting, 38(1/2), 145-164, 2011. DOI:10.1111/j.1468-5957.2010.02228.x

FELDMAN, M. S.; PENTLAND, B. T.Reconceptualizing Organizational Routines as a Source of Flexibility and Change. Administrative Science Quarterly, (1), 94-118, 2003. DOI:10.2307/3556620

FELDMAN, M. S. Organizational routines as a source of continuous change. Organization Science, 11(6), 611-629, 2000.

FELDMAN, M. S. A performative perspective on stability and change in organizational routines. Industrial and corporate change, 12(4), 727-752, ,2003.

FELDMAN, M. S. Routines as process: Past, present, and future. In HowardGrenville. J., Rerup, C., Langley, A. \& Tsoukas, H. Organizational Routines: How 


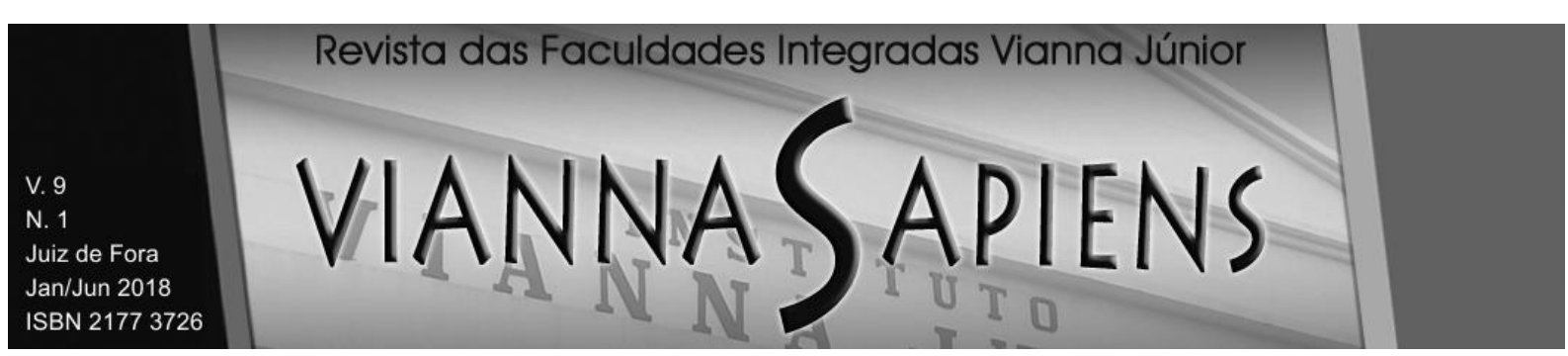

They Are Created, Maintained, and Changed. Oxford University Press, pp. 23-46. 2016. E-ISBN 978-0-231-53707-7

FELDMAN, M. S.; ORLIKOWSKI, W. J. Theorizing Practice and Practicing Theory. Organization Science, 22(5), 1240-1253, 2011. DOI:10.1287/orsc.1100.0612.

FELDMAN, M. S.; PENTLAND, B. T.; D'ADDERIO, L.; LAZARIC, N. Beyond routines as things: introduction to the special issue on routine dynamics. Organization Science, (3), 505-513, 2016. DOI:10.1287/orsc.2016.1070

FREZATTI, F. Beyond budgeting: inovação ou resgate de antigos conceitos do orçamento empresarial?. RAE-Revista de Administração de Empresas, 45(2), 2005.

FREZATTI, F.; NASCIMENTO, A. R. D.; JUNQUEIRA, E.; RELVAS, T. R. S. Processo orçamentário: uma aplicação da análise substantiva com utilização da grounded theory. Organizações \& Sociedade, 18(58), 445-466, 2011. doi:10.1590/S1984-92302011000300006

GIDDENS, A. The constitution of society: outline of the theory of structuration: University of California Press. 1984

GOMES, G.; LAVARDA, C. F.; TORRENS, E. W. Artigo - Finanças: Revisão da literatura sobre orçamento em cinco periódicos internacionais nos anos de 2000 até 2009. REGE - Revista de Gestão, 19(1) 107-123, 20121. doi:10.5700/rege454.

HANSEN, S. C.; VAN DER STEDE, W. A. Multiple facets of budgeting: an exploratory analysis. Management accounting research, v. 15, n. 4, 415-439, 2004. doi: 10.1016/j.mar.2004.08.001

HOPE, J.; FRASER, R. Beyond budgeting: how managers can break free from the annual performance trap. Harvard Business Press. Boston. MA., 2003a ISBN 9781-57851-866-1

HOPE, J.; FRASER, R. New Ways of Setting Rewards: The beyond budgeting model. California Management Review, 45(4), 104-119, 2003b. doi:

10.2307/41166190 


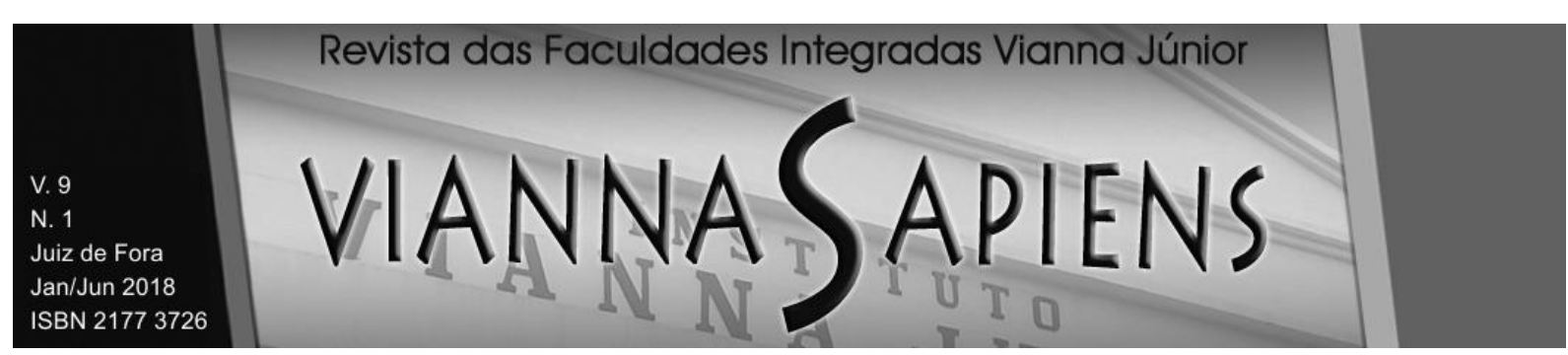

HOWARD-GRENVILLE. J.; RERUP, C.; LANGLEY, A.; TSOUKAS, H. Introduction: Advancing a Process Perspective on Routines by Zooming Out and Zooming In. In: Howard-Grenville. J., Rerup, C., Langley, A. \& Tsoukas, H. Organizational Routines: How They Are Created, Maintained, and Changed (Vol.5). Oxford University Press, 1-22, 2016.

HOWARD-GRENVILLE J.; RERUP C. A Process Perspective on Organizational Routines. In: Langley, A. \& Tsoukas, H. The SAGE Handbook of Process Organization Studies. Sage Publications Inc. p. 323-340. 2017.

KIHN, A. L. How do controllers and managers interpret budget targets? Journal of Accounting \& Organizational Change, v. 7, n. 3, 212-236, 2011.

LANGLEY, A.; TSOUKAS, $\mathrm{H}$. Introducing perspectives on process organization studies. Process, sensemaking, and organizing, 1(9), 1-27. United Kingdom, Europe : Oxford University Press. 2010

LEITE, R. M.; CHEROBIM, A. P. M. S.; SILVA, H. D. F. N.; BUFREM, L.S.

Orçamento empresarial: levantamento da produção científica no período de 1995 a 2006 / Business budget: a survey of scientific production in the period from 1995 to 2006. Revista Contabilidade \& Finanças, (47), 56, 2008. doi:10.1590/S151970772008000200006

LIBBY, T.; LINDSAY, R. M. Beyond budgeting or budgeting reconsidered? A survey of North-American budgeting practice. Management Accounting Research, 21(1) 56-75, 2010. DOI:10.1016/j.mar.2009.10.003

MENEGHETTI, F. K. O que é um ensaio-teórico?. RAC-Revista de Administração Contemporânea, 15(2), 2011.

MERCHANT, K. A. The Design of the Corporate Budgeting System: Influences on Managerial Behavior and Performance. The Accounting Review, 56(4), 813-829, 1981. Retrieved from http://www.jstor.org/stable/247203

MERCHANT, K. A. Modern management control systems: text and cases. Prentice Hall. 1998 


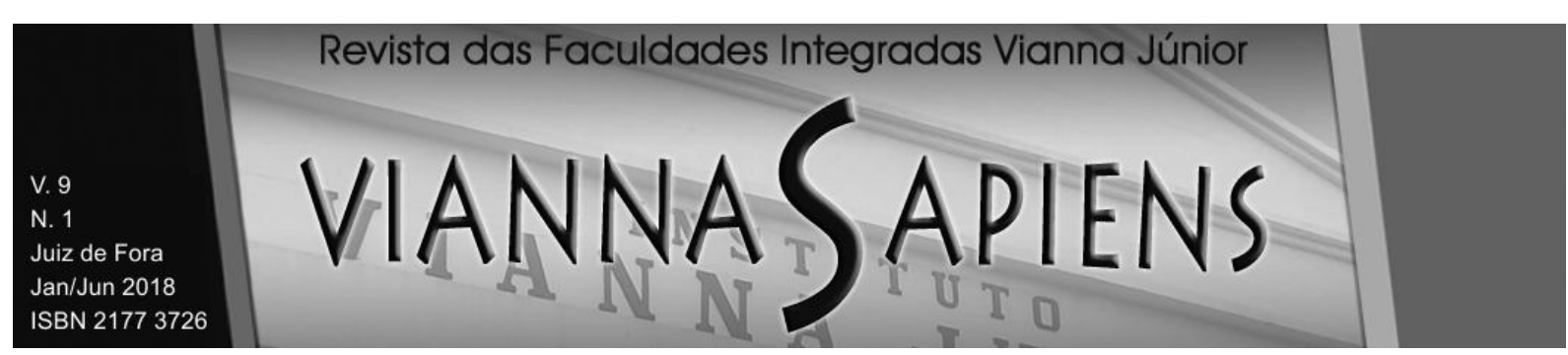

MUCCI, D. M.; FREZATTI, F.; DIENG, M. As múltiplas funções do orçamento empresarial. RAC - Revista de Administração Contemporânea, 20 (3), 283-304, 2016.

PADOVEZE, C. L.; TARANTO, F. C. Orçamento empresarial: novos conceitos e técnicas. São Paulo: Pearson Prentice Hall, 2009. ISBN: 978857605178

PARMIGIANI, A.; HOWARD-GRENVILLE, J.. Routines revisited: Exploring the capabilities and practice perspectives. Academy of Management Annals, 5(1), 413453, 2011. DOI: https://doi.org/10.1080/19416520.2011.589143

PENTLAND, B. T.; RUETER, H. H. Organizational routines as grammars of action. Administrative Science Quarterly, 484-510, 1994. DOI:10.2307/2393300

PIETRZAK, Z. Traditional versus Activity-based Budgeting in Non-manufacturing Companies. Social Sciences (1392-0758), 82(4), 26-37, 2013.

DOI:10.5755/j01.ss.82.4.6604

REKA, C. I.; ŞTEFAN, P.; DANIEL, C. V. Traditional budgeting versus beyond budgeting: a literature review. Annals of the University of Oradea, Economic Science Series, 23(1) 573-581, 2014.

RERUP, C.; FELDMAN, M. S. Routines as a source of change in organizational schemata: the role of trial-and-error learning. Academy of Management Journal, 54(3), 577-610, 2011. DOI:10.5465/AMJ.2011.61968107

SPONEM, S.; LAMBERT, C. Pratiques budgétaires, rôles et critiques du budget. Perception des DAF et des contrôleurs de gestion. Comptabilité-Contrôle-Audit, v. 16, n. 1, 159-194, 2010. doi:10.3917/cca.161.0159

VAN DER STEDE, W. A. The relationship between two consequences of budgetary controls: budgetary slack creation and managerial short-term orientation.

Accounting, Organizations and Society, 25(6) 609-622, 2000.

DOI:10.1016/S0361-3682(99)00058-6

Recebido em 11/05/2018

Publicado em 13/08/2018 